\title{
ORBITAL SOLUTIONS AND ABSOLUTE ELEMENTS OF THE ECLIPSING BINARY MY CYGNI
}

\author{
Rebecca S. Tucker ${ }^{1,2}$, James R. Sowell ${ }^{1}$, Richard M. Williamon ${ }^{3}$, and Jeffrey L. Coughlin ${ }^{3,4}$ \\ ${ }^{1}$ School of Physics, Georgia Institute of Technology, Atlanta, GA 30332, USA; jim.sowell@ physics.gatech.edu \\ ${ }^{2}$ Department of Physics, California Institute of Technology, Pasadena, CA 91125, USA \\ ${ }^{3}$ Department of Physics, Emory University, Atlanta, GA 30322, USA; rwilliamon@physics.emory.edu \\ ${ }^{4}$ Department of Astronomy, New Mexico State University, Las Cruces, NM 88003, USA \\ Received 2007 July 13; accepted 2008 October 3; published 2009 January 13
}

\begin{abstract}
Differential $U B V$ photoelectric photometry for the eclipsing binary MY Cyg is presented. The WilsonDevinney program is used to simultaneously solve the three light curves together with previously published radial velocities. A comparison is made with the previous solution found with the Russell-Merrill method. We examine the long-term apsidal motion of this well-detached, slightly eccentric system. We determine absolute dimensions, discuss metallicity/Am-star issues, and estimate the evolutionary status of the stars.
\end{abstract}

Key words: binaries: close - binaries: eclipsing - binaries: spectroscopic

Online-only material: color figures, machine-readable and VO tables

\section{INTRODUCTION}

The variability of MY Cygni (HD 193637, BD +33 3862 , SAO 69850) was first noted by Hoffmeister (1930). From a study of Bamberg and Sonneberg plates, Rugemer (1932) found a period of 2 days and noted the absence of a secondary eclipse. Rough photographic light curves based on the 2 day period have been given by Wachmann (1948), Gasposchkin (1953), Zessewitsch (1954), and Filatov (1963). The correct 4 day period was discovered from spectroscopic observations by Popper (1969), who also pointed out the double-line nature of the system. Later work by Popper (1971) included a spectroscopic analysis, indicating components of almost equal mass, and a discussion of the Am metallic-line characteristics. Williamon (1975) noted that the very similar primary and secondary eclipses had been reversed in the work by Popper (1971). With this correction, Popper \& Etzel (1981) presented a solution of their photometric data.

This paper is a re-examination of the photometric data and the orbital solution by Williamon (1975). His results were obtained with the Russell-Merrill method on 16-point normals outside of eclipse and 4-point normals inside eclipse. In contrast, the Wilson-Devinney (WD) binary software can analyze each data point (HJD and $\Delta$ mag) and can solve the light curves simultaneously. MY Cyg has a nonzero eccentricity, and its components are relatively close, so tidal deformations could exist; both of these aspects of the system are handled well by the WD software. With the inclusion of previously published radial velocities, our solution with the WD program is a more accurate description of the orbit and absolute dimensions.

\section{OBSERVATIONS}

The photometric measurements of MY Cyg were obtained with the 36 inch Cassegrain reflector at the Fernbank Science Center Observatory (Atlanta, GA) during 1972, 1973, and 1974. A total of 1733 observations were made in each color with the standard $U B V$ filters of the Johnson-Morgan (1953) system combined with an unrefrigerated EMI 6256s photomultiplier. The observations were recorded with a Honeywell strip-chart recorder, and deflections were read with a $5 \mathrm{~s}$ timing accuracy. All observations of MY Cyg were made differentially with respect to the comparison star $\mathrm{BD}+33^{\circ} 3850$ (SAO 69818), and they were corrected for atmospheric extinction by means of nightly extinction coefficients determined from the comparison star via the technique of Hardie (1962). The Heliocentric Julian Dates and differential magnitudes for all of the observations are given in Table 1. Measurements of the check star BD $+32^{\circ} 3788$ (SAO 69840) were obtained on many nights with no indication of any variability of the comparison star. For completeness, the radial velocities of Popper (1971) are provided in Table 2.

\section{LIGHT AND VELOCITY SOLUTIONS}

Light and velocity solutions were obtained with the latest version of the WD program. The program's physical model is described in detail in Wilson \& Devinney (1971) and Wilson $(1979,1990)$. The program now includes an improved stellar atmosphere treatment (Van Hamme \& Wilson 2003) that is based on prefitted Legendre functions to Kurucz (1993) atmosphere models.

We made simultaneous $U B V$ light and double-lined radial velocity (RV) solutions to improve parameter consistency (Wilson 1979; Van Hamme \& Wilson 1984, 1985). Radial velocities were those of Popper (1971). Curve-dependent weights were based on standard deviations that are listed in Table 3; light level-dependent weights were applied inversely proportional to the square root of the light level. A square-root limb darkening law with coefficients $x, y$ from Van Hamme (1993) was adopted, and the detailed reflection treatment of Wilson (1990) was used with only a single reflection. Gravity darkening $(g)$ and bolometric albedo $(A)$ coefficients were fixed at canonical values for stars with radiative outer layers (Lucy 1967).

Due to the lack of accurate spectral classifications, the determination of the surface temperatures was difficult. Popper (1971) gave spectral types for the two components of A7 and A5 based on the Ca II K line and F2 for both stars from their metallic lines. These spectral types correspond to a temperature range from 8180 to $7000 \mathrm{~K}$ (Cox 2000). We allowed the primary's temperature $\left(T_{1}\right)$ to vary in $100 \mathrm{~K}$ increments from 6700 to $8200 \mathrm{~K}$. Appropriate limb darkening and bolometric coefficients were used, and solutions were found at each incremental temperature. Unfortunately, there was miniscule change in the rms results, so the WD program was not able to 
Table 1

MY Cyg Photometric Observations

\begin{tabular}{lllllll}
\hline \hline HJD-2400000.0 & $\Delta V$ & HJD-2400000.0 & $\Delta B$ & HJD-2400000.0 & $\Delta U$ \\
\hline 41561.6326 & -0.281 & 41561.6350 & -0.006 & 41561.6360 & +0.111 \\
41561.7442 & -0.804 & 41561.7451 & -0.468 & 41561.7460 & -0.376 \\
41561.7483 & -0.796 & 41561.7490 & -0.497 & 41561.7499 & -0.404 \\
41561.7592 & -0.793 & 41561.7600 & -0.475 & 41561.7606 & -0.384 \\
41561.7720 & -0.816 & 41561.7720 & -0.475 & 41561.7728 & -0.385 \\
\hline
\end{tabular}

(This table is available in its entirety in machine-readable and Virtual Observatory (VO) forms in the online journal. A portion is shown here for guidance regarding its form and content.)

Table 2

MY Cyg Radial Velocities ${ }^{\mathrm{a}}$

\begin{tabular}{lrr}
\hline \hline HJD-2400000.0 & RV Primary & RV Secondary \\
\hline 38538.970 & +43.7 & -144.7 \\
39687.893 & +37.6 & -144.6 \\
39691.938 & +40.7 & -150.9 \\
39692.839 & +5.9 & -111.2 \\
39717.776 & -132.7 & +28.4 \\
39718.814 & -112.1 & +4.3 \\
39719.793 & +30.2 & -126.9 \\
39757.732 & -121.9 & +22.8 \\
39783.635 & -2.7 & -104.8 \\
39784.619 & +38.1 & -137.4 \\
39812.603 & +42.5 & -151.1 \\
39818.584 & -147.9 & +40.1 \\
39961.016 & +18.6 & -129.8 \\
39962.008 & -127.5 & +15.6 \\
40019.949 & -3.1 & -107.7 \\
40022.965 & -144.5 & +33.1 \\
40108.653 & +47.7 & -154.1 \\
40132.690 & +50.3 & -153.5 \\
40132.713 & +45.4 & -153.0 \\
40134.656 & -158.8 & +44.3 \\
40134.694 & -158.3 & +48.3 \\
40170.575 & -146.3 & +43.7 \\
40170.595 & -148.7 & +45.4 \\
40376.930 & +48.2 & -159.5 \\
40406.877 & -151.1 & +39.4 \\
40406.899 & -153.4 & +41.6 \\
40428.856 & +41.6 & -144.3 \\
40849.717 & +47.9 & -153.6 \\
\hline & &
\end{tabular}

Note. ${ }^{a}$ These radial velocities were previously published by Popper (1971) and are provided here for convenience and completeness of our orbital solution.

satisfactorily distinguish the best set of temperatures. The use of supplemental information was necessary. First, Popper (1971) stated that the photometric indices of MY Cyg were "among the later Am stars." Second, the $B_{2}-V_{1}$ temperature index from the Geneva photometry by Hauck \& Curchod (1980) equals +0.124 , which corresponds to a spectral type of F0 V or F0 III for normal stars (Hauck 1994). Third, in their study of the galactic distributions of the HD stars in the Michigan Spectral Catalogue (Caballero-Nieves et al. 2007), the Am stars were shown to have distributions similar to the early F dwarfs and giants. Returning to the rms results and the temperatures of Cox for early dwarfs, the primary's temperature was set at $7100 \mathrm{~K}$. We estimate the external uncertainty in $T_{1}$ to be approximately $\pm 200 \mathrm{~K}$; consequently, the derived $T_{2}$ value would have a similar standard deviation. Solution parameters and standard errors are presented in Table 4.

The two components have almost identical absolute dimensions, which are listed in Table 5. The masses $\left(M_{1}=\right.$
Table 3

Characteristic Measurement Errors for MY Cyg Data

\begin{tabular}{lc}
\hline \hline Curve & $\sigma^{\mathrm{a}}$ \\
\hline RV Primary & $3.6 \mathrm{~km} \mathrm{~s}^{-1}$ \\
RV Secondary & $2.8 \mathrm{~km} \mathrm{~s}^{-1}$ \\
$V$ & 0.012 \\
$B$ & 0.014 \\
$U$ & 0.015 \\
\hline
\end{tabular}

Note. ${ }^{\text {a }}$ For the light curves, in units of total light at phase 0.25 .

Table 4

Light and Velocity Curve Results ${ }^{\mathrm{a}}$

\begin{tabular}{lcc}
\hline \hline Parameter & Symbol & Value \\
\hline Inclination $\left(^{\circ}\right)$ & $i$ & $88.58 \pm 0.02$ \\
Surface potential & $\Omega_{1}$ & $8.373 \pm 0.032$ \\
Surface potential & $\Omega_{2}$ & $8.128 \pm 0.042$ \\
Mass ratio & $M_{2} / M_{1}$ & $0.994 \pm 0.004$ \\
Temperature (K) & $T_{1}$ & $7100^{\mathrm{b}}$ \\
Temperature $(\mathrm{K})$ & $T_{2}$ & $7036 \pm 4$ \\
Semimajor axis $\left(R_{\odot}\right)$ & $a$ & $16.30 \pm 0.08$ \\
Eccentricity & $e$ & $0.010 \pm 0.001$ \\
Longitude of periastron $\left(^{\circ}\right)$ & $\omega$ & $69.6 \pm 2.4$ \\
System velocity $\left(\mathrm{km} \mathrm{s}^{-1}\right)$ & $V_{\gamma}$ & $-53.50 \pm 0.42$ \\
Orbital velocity $\left(\mathrm{km} \mathrm{s}^{-1}\right)$ & $K_{1}$ & $102.4 \pm 3.7$ \\
Orbital velocity $\left(\mathrm{km} \mathrm{s}^{-1}\right)$ & $K_{2}$ & $103.0 \pm 2.4$ \\
Luminosity ratio & $L_{1} /\left(L_{1}+L_{2}\right)_{V}$ & $0.4947 \pm 0.0040$ \\
Luminosity ratio & $L_{1} /\left(L_{1}+L_{2}\right)_{B}$ & $0.4973 \pm 0.0039$ \\
Luminosity ratio & $L_{1} /\left(L_{1}+L_{2}\right)_{U}$ & $0.4978 \pm 0.0039$ \\
Limb darkening $($ bolo $)$ & $x_{1}, y_{1}$ & $+0.082,+0.643$ \\
Limb darkening $($ bolo $)$ & $x_{2}, y_{2}$ & $+0.086,+0.638$ \\
Limb darkening $(V)$ & $x_{1}, y_{1}$ & $+0.053,+0.733$ \\
Limb darkening $(B)$ & $x_{1}, y_{1}$ & $+0.171,+0.712$ \\
Limb darkening $(U)$ & $x_{1}, y_{1}$ & $+0.073,+0.826$ \\
Limb darkening $(V)$ & $x_{2}, y_{2}$ & $+0.063,+0.724$ \\
Limb darkening $(B)$ & $x_{2}, y_{2}$ & $+0.191,+0.691$ \\
Limb darkening $(U)$ & $x_{2}, y_{2}$ & $+0.088,+0.817$ \\
Albedo (bolo) & $A_{1}, A_{2}$ & $0.5,0.5$ \\
Gravity darkening & $g_{1}, g_{2}$ & $0.3,0.3$ \\
Rotation/orbit ratio & $F_{1}, F_{2}$ & $1.0,1.0$ \\
\hline & &
\end{tabular}

Notes.

a WD simultaneous solution, including proximity effects, of the light and velocity data.

${ }^{\mathrm{b}}$ Based on the estimated F2 V spectral type; see Section 3.

$\left.1.82 \pm 0.03 M_{\odot}, M_{2}=1.80 \pm 0.03 M_{\odot}\right)$ are similar to those obtained by Williamon $\left(M_{1}=1.81 M_{\odot}, M_{2}=1.78 M_{\odot}\right)$. Our $\operatorname{radii}\left(R_{1}=2.22 \pm 0.02 R_{\odot}, R_{2}=2.28 \pm 0.02 R_{\odot}\right)$ are in excellent agreement with Williamon's values of $R_{1}=2.21 R_{\odot}$ and $R_{2}=2.29 R_{\odot}$. Figures $1-3$ show the observed measurements and fitted light curves in each bandpass. The light curves are very clean and there is no visual evidence for cool or hot spots on the surface of either component. Inspection of residual plots of the $U B V$ data with the theoretical curves shows no asymmetries. The radial velocities of Popper (1971) are shown in Figure 4, with the computed curve corresponding to the simultaneous light-velocity solution. Popper's data were obtained at orbital phases well outside eclipses and are essentially unaffected by the Rossiter effect.

A previous solution, found independently of Williamon, was performed by Tremko et al. (1978) using the direct interative minimization method of Horak (1966). Their computed masses of $M_{1}=1.81 \pm 0.03 M_{\odot}$ and $M_{2}=1.79 \pm 0.03 M_{\odot}$ are very similar to ours, but their radii of $R_{1}=2.26 \pm 0.08 R_{\odot}$ and 


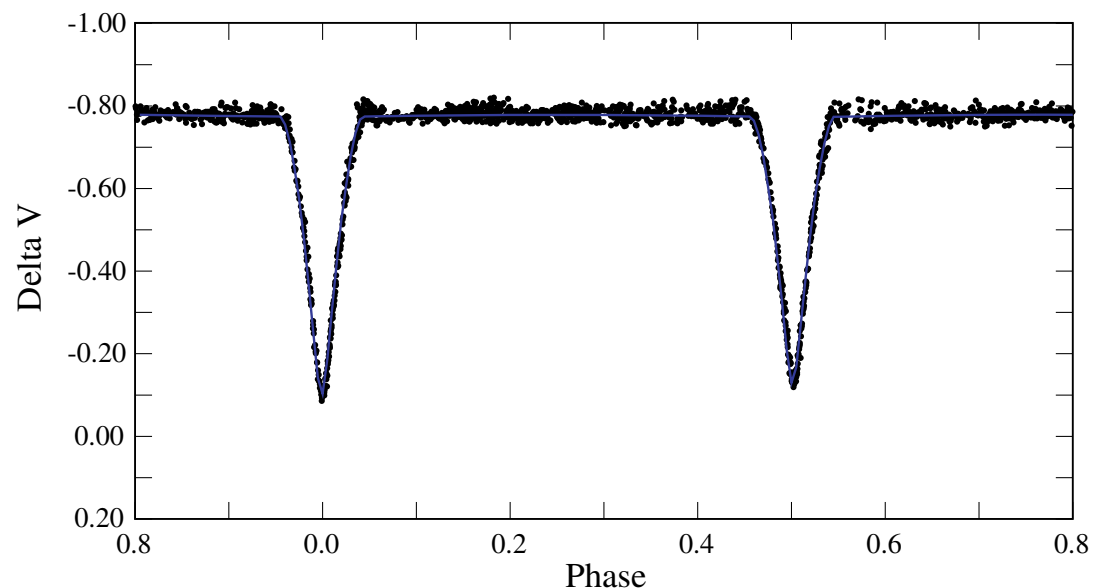

Figure 1. Differential $V$ magnitudes of MY Cyg plotted with the computed solution curve and the primary eclipse ephemeris. (A color version of this figure is available in the online journal.)

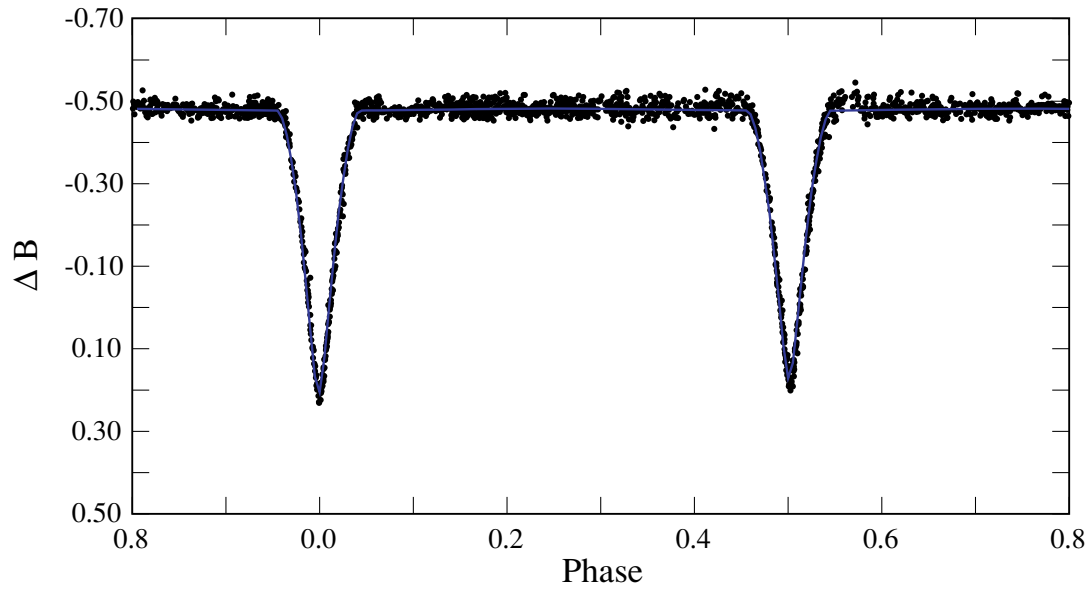

Figure 2. Differential $B$ magnitudes of MY Cyg plotted with the computed solution curve and the primary eclipse ephemeris.

(A color version of this figure is available in the online journal.)

Table 5

Magnitudes $^{\mathrm{a}}$ and Absolute Dimensions

\begin{tabular}{lcc}
\hline \hline Parameter & Primary & Secondary \\
\hline$V$ & 9.10 & 9.08 \\
$B$ & 9.38 & 9.37 \\
$U$ & 9.52 & 9.51 \\
$M\left(M_{\odot}\right)$ & $1.82 \pm 0.03$ & $1.80 \pm 0.03$ \\
$R\left(R_{\odot}\right)$ & $2.22 \pm 0.02$ & $2.28 \pm 0.02$ \\
$\log \left(L / L_{\odot}\right)$ & $1.05 \pm 0.04$ & $1.06 \pm 0.05$ \\
$\mathrm{M}_{\text {bol }}$ & $2.12 \pm 0.09$ & $2.10 \pm 0.10$ \\
$\log g\left(\mathrm{~cm} \mathrm{~s}^{-2}\right)$ & $4.01 \pm 0.01$ & $3.98 \pm 0.01$ \\
\hline
\end{tabular}

Note. ${ }^{a}$ Based on $V=8.34, B-V=0.28$, and $U-B=0.14$ (outside of eclipse) from Tremko et al. (1978), and on our luminosity ratios.

$R_{2}=2.15 \pm 0.08 R_{\odot}$ are reversed. They used rather similar temperatures of $T_{1}=7160 \mathrm{~K}$ and $T_{2}=7140 \mathrm{~K}$. Though Tremko et al. have substantial differential $U B V$ measurements, their phase coverage was incomplete and their observations had larger residuals, so these data were not included in our solution. The Tremko et al. data are plotted with our final-solution light curves in Figures 5-7, and visual inspection shows that our solution fits their data quite well.

The WD program provides geometrical information about the two stars. Relative radii are given in four directions: from the center toward the poles, toward the sides, toward the back (i.e.,
Table 6

Model Radii

\begin{tabular}{lc}
\hline \hline Parameter & Value \\
\hline$r_{1}$ (pole) & $0.1355 \pm 0.0006$ \\
$r_{1}$ (point) & $0.1365 \pm 0.0006$ \\
$r_{1}$ (side) & $0.1359 \pm 0.0006$ \\
$r_{1}$ (back) & $0.1364 \pm 0.0006$ \\
$\left\langle r_{1}\right\rangle^{\mathrm{a}}$ & $0.1359 \pm 0.0006$ \\
$\left\langle r_{1}\right\rangle /\left\langle r_{1}\right\rangle_{\text {lobe }}$ & $0.3602 \pm 0.0017$ \\
\hline$r_{2}$ (pole) & $0.1395 \pm 0.0008$ \\
$r_{2}$ (point) & $0.1406 \pm 0.0009$ \\
$r_{2}$ (side) & $0.1399 \pm 0.0008$ \\
$r_{2}$ (back) & $0.1404 \pm 0.0009$ \\
$\left\langle r_{2}\right\rangle^{\mathrm{a}}$ & $0.1400 \pm 0.0006$ \\
$\left\langle r_{2}\right\rangle /\left\langle r_{2}\right\rangle_{\text {lobe }}$ & $0.3719 \pm 0.0026$ \\
\hline
\end{tabular}

Note. a "Equal-volume" mean radii.

away from the companion), and toward the inner Lagrangian point, L1. In addition, it computes "equal-volume" mean radii $(\langle r\rangle)$ and the percentage of the Roche lobe $\left(\langle r\rangle /\langle r\rangle_{\text {lobe }}\right)$ that is filled. These quantities are given in Table 6 . For both components the four directional radii are basically equal, so the stars are spherically shaped. The Roche lobes are 36\% and 37\% filled, respectively, so even though MY Cyg is a rather close binary, it is a well detached system. 


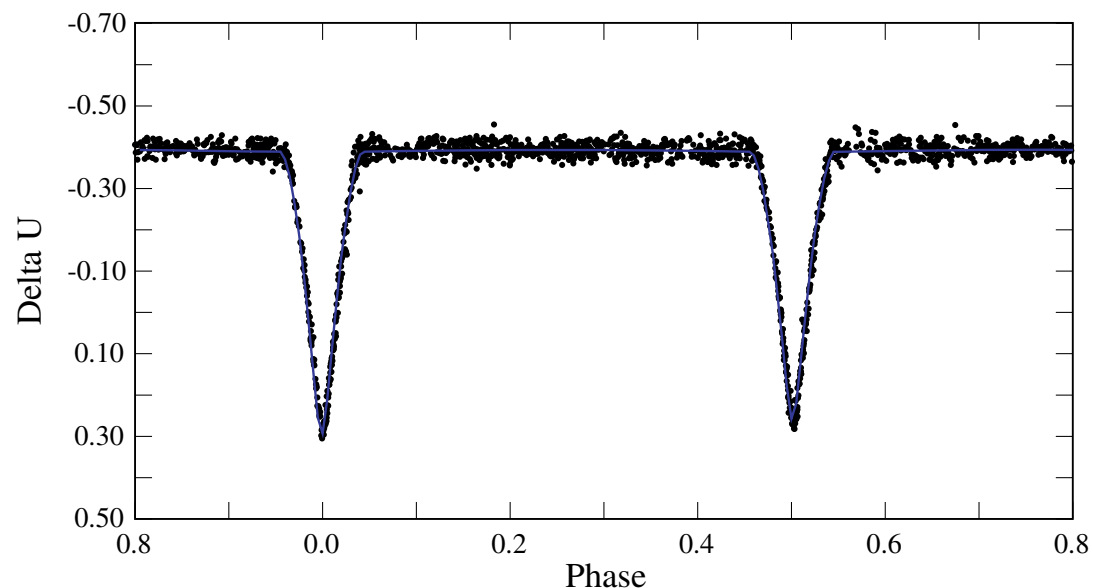

Figure 3. Differential $U$ magnitudes of MY Cyg plotted with the computed solution curve and the primary eclipse ephemeris.

(A color version of this figure is available in the online journal.)

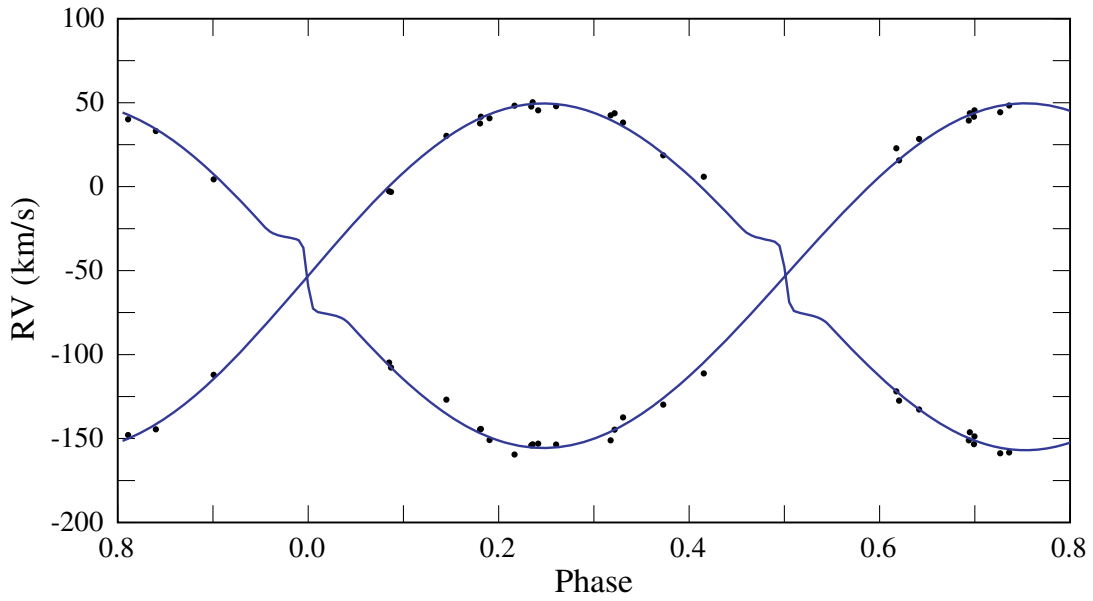

Figure 4. MY Cyg radial velocities of Popper (1971) and computed curves for the simultaneous light and velocity solution including the Rossiter proximity effects.

(A color version of this figure is available in the online journal.)

\section{EPHEMERIS PARAMETERS AND APSIDAL MOTION}

The interesting aspect of the MY Cyg system is its slightly eccentric orbit, which causes the secondary minima to be displaced from phase 0.500 in the light curve. Eccentric orbits also experience apsidal motion, the gradual displacement of its longitude of periastron, due to both the classical effects of gravitational tidal forces as well as general relativistic effects. Thus, MY Cyg provides a means to observationally test the theoretical predictions of classical gravitational effects and general relativity.

A modern light curve was obtained by Coughlin (2007) expressly for measuring the current displacement of the secondary eclipse. He obtained differential BVRI photometric measurements on three nights, and used the same comparison star as Williamon (1975). His primary eclipse coverage began after first contact and ended slightly past mid-eclipse. Secondary eclipse measurements included first contact and the central region of mid-eclipse. His observations are reproduced in Table 7 . We had hoped that there was sufficient Coughlin data and phase coverage for the WD program to accurately analyze the combined Williamon and Coughlin data. We re-ran the WD program numerous times, in some cases allowing all of the parameters to vary while in others just the orbital ones, such as the period, $\omega$, $d \omega / d t$, and eccentricity. However, we were not satisfied with the results, residuals, and residual plots of the combined data, and thus the Coughlin data were only used for the improved ephemeris and apsidal motion study.

In the first detailed analysis of the system, Williamon (1975) recognized that the secondary had been displaced from phase 0.50 over the 45 years prior to his study and speculated that it was due to apsidal motion. Williamon found secondary minimum at phase 0.5022 , and he derived $e=0.010$ and $\omega=69^{\circ} .6$. Our reanalysis found indentical values of $e=0.010 \pm 0.001$ and $\omega=69.6 \pm 2.4$. Again it is seen that for MY Cyg the RussellMerrill and WD solutions are equivalent.

The time of minimum (TOM) for both eclipses as determined by Williamon (1975) and Coughlin (2007) are in Table 8. Beginning with the previously mentioned ephemeris, we performed a linear least-squares analysis on the two sets of TOM data and obtained the following ephemerii:

$$
\begin{aligned}
\text { Minimumlight }(\text { Pri })= & 2,441,585.62896 \pm 0.00021 \\
& +4.005187081 \pm 0.000000133 E,
\end{aligned}
$$

and

$$
\begin{aligned}
\text { Minimumlight }(\operatorname{Sec})= & 2,441,587.64061 \pm 0.00019 \\
& +4.005188234 \pm 0.000000130 E,
\end{aligned}
$$

where the "errors" are the uncertainties of the parameters. 


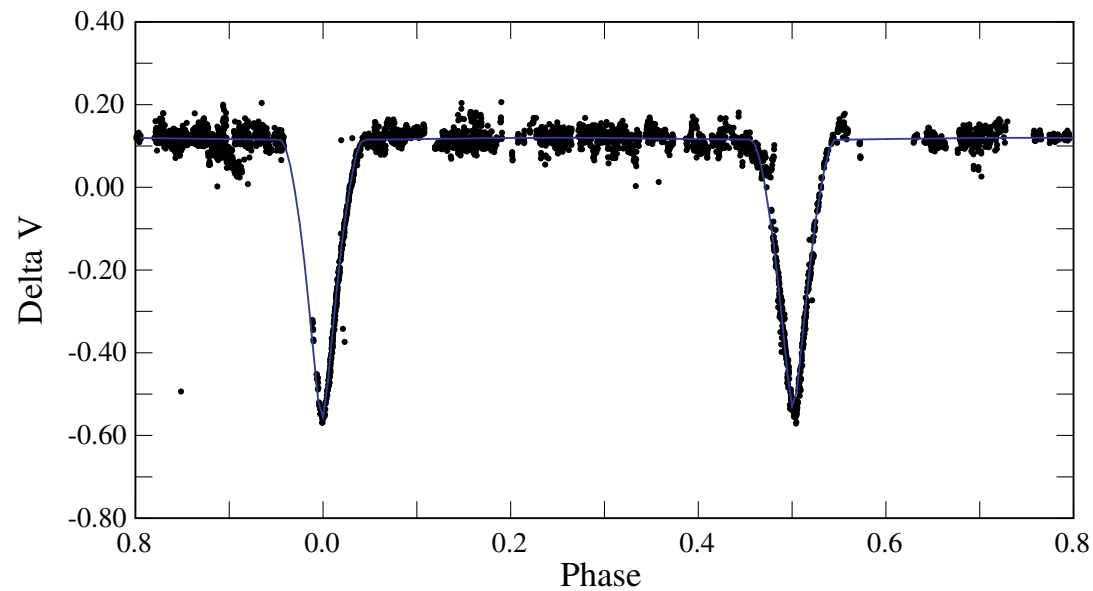

Figure 5. Differential $V$ magnitudes of MY Cyg from Tremko et al. (1978) plotted with the computed solution curve and the primary eclipse ephemeris. (A color version of this figure is available in the online journal.)

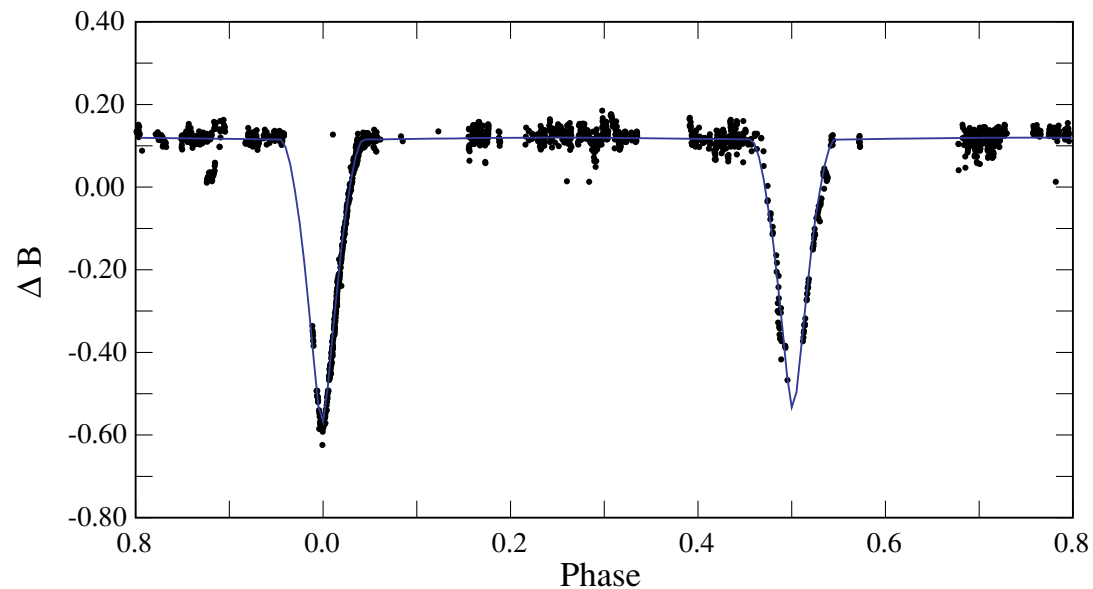

Figure 6. Differential $B$ magnitudes of MY Cyg from Tremko et al. (1978) plotted with the computed solution curve and the primary eclipse ephemeris. (A color version of this figure is available in the online journal.)

Apsidal motion is recognized by the displacement of secondary eclipse from phase 0.500 . The displacement, $D$, is calculated by

$$
D=[(\text { Time }(\text { Sec })-\text { Time }(\text { Pri }))-((\text { Cycles }+0.5) * \text { Period })],
$$

where Cycles is an integer. The displacements for each of the secondary minima were computed with the primary eclipse ephemeris. The displacements are related to the longitudes of periastron $(\omega)$ by the formula (Guinan \& Maloney 1985)

$$
D=\frac{P}{\pi}\left[\arctan \left(\frac{e \cos \omega}{\left(1-e^{2}\right)^{1 / 2}}\right)+\frac{e \cos \omega}{1-e^{2} \sin ^{2} \omega}\left(1-e^{2}\right)^{1 / 2}\right]
$$

The computed $D$ and $\omega$ values (using $e=0.010$ ) are given in Table 8. Taking averages for the data sets, the derived $d \omega / d t$ is $(-0.21 \pm 0.07) \mathrm{yr}^{-1}$. Although the individually computed longitudes are higher for the Williamon data than those computed by the WD program and by Williamon (1975), using tables from Irwin (1962), it is only the difference (i.e., $d \omega / d t)$ that matters.

Theoretically, the total apsidal motion is due to two components: the classical, which arises from tidal interactions on the oblate stars, and the relativistic, which is a direct consequence of Einstein's Theory of General Relativity. The classical part is calculated by (Sterne 1939)

$$
\begin{aligned}
\frac{d \omega}{d t}(c l) & =365.25\left(\frac{360}{P}\right)\left\{k _ { 2 , 1 } r _ { 1 } ^ { 5 } \left[15 f_{2}(e)\left(\frac{M_{2}}{M_{1}}\right)+\left(\frac{\omega_{r, 1}}{\omega_{k}}\right)^{2}\right.\right. \\
& \left.\times\left(\frac{1+M_{2} / M_{1}}{\left(1-e^{2}\right)^{2}}\right)\right]+k_{2,2} r_{2}^{5}\left[15 f_{2}(e)\left(\frac{M_{1}}{M_{2}}\right)\right. \\
& \left.\left.+\left(\frac{\omega_{r, 2}}{\omega_{k}}\right)^{2}\left(\frac{1+M_{1} / M_{2}}{\left(1-e^{2}\right)^{2}}\right)\right]\right\},
\end{aligned}
$$

where

$$
f_{2}(e)=\left(1+\frac{3}{2} e^{2}+\frac{1}{8} e^{4}\right)\left(1-e^{2}\right)^{-5}
$$

$P$ is the period in days, $M_{1}$ and $M_{2}$ are the masses in solar masses, $k_{2,1}$ and $k_{2,2}$ are known as the apsidal motion constants of each component, $r_{1}$ and $r_{2}$ are the fractional radii, $\omega_{r, 1}$ and $\omega_{r, 2}$ are the angular rotation speeds, and $\omega_{k}$ is the mean angular Keplerian velocity, equal to $2 \pi / P$.

Values of $k_{2}$ based on computations of main-sequence stellar interiors are provided in Table 1 of Jeffery (1984). Using his quantities for $2 M_{\odot}$ stars with our $\log g=4.0$ gives $k_{2,1}=k_{2,2}=0.00400$. Popper (1971) makes no mention of any line broadening or rotational velocities, and thus we may assume the stars are tidally locked so that $\omega_{r, 1} / \omega_{k}=\omega_{r, 2} / \omega_{k}=1$. 


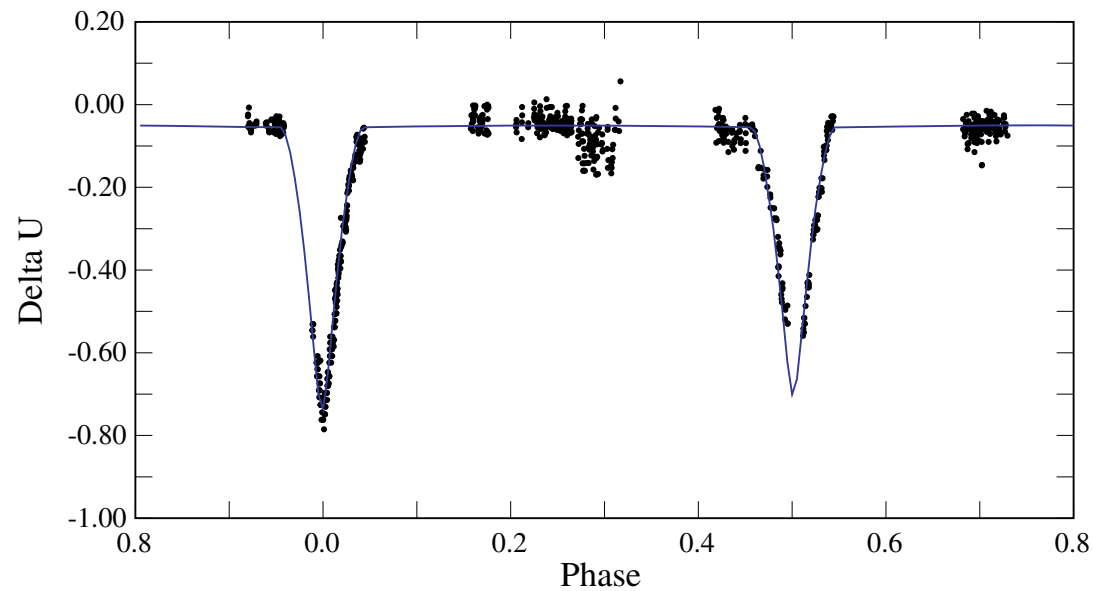

Figure 7. Differential $U$ magnitudes of MY Cyg from Tremko et al. (1978) plotted with the computed solution curve and the primary eclipse ephemeris. (A color version of this figure is available in the online journal.)

Table 7

MY Cyg Photometric Observations by Coughlin ${ }^{\mathrm{a}}$

\begin{tabular}{lccccccc}
\hline \hline HJD-2400000.0 & $\Delta B$ & HJD-2400000.0 & $\Delta V$ & HJD-2400000.0 & $\Delta R$ & HJD-2400000.0 & $\Delta I$ \\
\hline 54029.6135 & -2.626 & 54029.6139 & -1.703 & 54029.6160 & -1.185 & 54029.6146 & -0.639 \\
54029.6152 & -2.606 & 54029.6157 & -1.703 & 54029.6176 & -1.194 & 54029.6163 & -0.627 \\
54029.6168 & -2.608 & 54029.6173 & -1.679 & 54029.6191 & -1.148 & 54029.6179 & -0.629 \\
54029.6184 & -2.599 & 54029.6188 & -1.695 & 54029.6207 & -1.157 & 54029.6194 & -0.615 \\
54029.6199 & -2.593 & 54029.6204 & -1.683 & 54029.6222 & -1.125 & 54029.6210 & -0.627 \\
\hline
\end{tabular}

(This table is available in its entirety in machine-readable and Virtual Observatory (VO) forms in the online journal. A portion is shown here for guidance regarding its form and content.)

Table 8

Times of Minima ${ }^{\mathrm{a}}$

\begin{tabular}{|c|c|c|c|c|c|c|c|c|c|}
\hline HJD & Band & Epoch & $\mathrm{O}-\mathrm{C}$ & HJD & Band & Epoch & $\mathrm{O}-\mathrm{C}$ & $\mathrm{D}$ & $\omega$ \\
\hline & \multicolumn{3}{|c|}{ Primary eclipse } & & \multicolumn{3}{|c|}{ Secondary eclipse } & & \\
\hline 41585.6287 & $B$ & 0.0 & -0.00026 & 41563.6110 & V & -5.5 & +0.00152 & 0.0106 & 71.0 \\
\hline 41585.6289 & V & 0.0 & -0.00006 & 41563.6109 & $B$ & -5.5 & +0.00142 & 0.0105 & 71.2 \\
\hline 41585.6303 & $U$ & 0.0 & +0.00134 & 41563.6097 & $U$ & -5.5 & +0.00022 & 0.0093 & 73.4 \\
\hline 41601.6503 & $V$ & 4.0 & +0.00059 & 41599.6543 & $V$ & 3.5 & -0.00187 & 0.0072 & 77.2 \\
\hline 41601.6503 & $B$ & 4.0 & +0.00059 & 41599.6565 & $B$ & 3.5 & +0.00033 & 0.0094 & 73.2 \\
\hline 41601.6515 & $U$ & 4.0 & +0.00179 & 41599.6562 & $U$ & 3.5 & +0.00003 & 0.0091 & 73.8 \\
\hline 42302.5575 & & 179.0 & +0.00005 & 41603.6600 & & 4.5 & -0.00136 & 0.0077 & 76.3 \\
\hline 42306.5623 & & 180.0 & -0.00033 & 42320.5894 & $V$ & 183.5 & -0.00066 & 0.0086 & 74.6 \\
\hline 42338.6030 & $B$ & 188.0 & -0.00113 & 42320.5900 & $B$ & 183.5 & -0.00006 & 0.0092 & 73.5 \\
\hline 42338.6033 & $V$ & 188.0 & -0.00083 & 42320.5891 & $U$ & 183.5 & -0.00096 & 0.0083 & 75.2 \\
\hline 42338.6042 & $U$ & 188.0 & +0.00007 & 42324.5950 & V & 184.5 & -0.00025 & 0.0090 & 73.9 \\
\hline \multirow[t]{3}{*}{42378.6539} & & 198.0 & -0.00210 & 42324.5957 & $B$ & 184.5 & +0.00045 & 0.0097 & 72.6 \\
\hline & & & & 42324.5933 & $U$ & 184.5 & -0.00195 & 0.0073 & 77.0 \\
\hline & & & & 42372.6586 & & 196.5 & +0.00110 & 0.0104 & 71.4 \\
\hline 54029.7470 & $B$ & 3107.0 & +0.00176 & 54031.7616 & $I$ & 3107.5 & +0.00119 & 0.0138 & 64.8 \\
\hline 54029.7446 & V & 3107.0 & -0.00064 & 54031.7616 & $V$ & 3107.5 & +0.00113 & 0.0138 & 64.9 \\
\hline 54029.7444 & $R$ & 3107.0 & -0.00077 & 54031.7591 & $R$ & 3107.5 & -0.00136 & 0.0113 & 69.7 \\
\hline 54029.7451 & $I$ & 3107.0 & -0.00010 & 54031.7595 & $B$ & 3107.5 & -0.00093 & 0.0117 & 68.9 \\
\hline
\end{tabular}

Note. ${ }^{\text {a }}$ Times of minima, residuals, displacements, and $\omega$ 's with respect to the linear, least-squares ephemerii for the primary and secondary eclipses, respectively, as given in Section 4 . The $D$ (displacement) values are computed with the primary eclipse ephemeris. The upper set of data are from Williamon (1975) and the lower are from Coughlin (2007).

Our orbital solution in Section 3 found $M_{1}=1.82 M_{\odot}$, $M_{2}=1.80 M_{\odot}, r_{1}=0.136$, and $r_{2}=0.140$. Using $e=0.010$ and $P=4.005186427$ days as before, the rate of change of the longitude of periastron due to tidal interactions is $-0.22 \mathrm{yr}^{-1}$.

The equation for the relativistic contribution is

$$
\frac{d \omega}{d t}(g r)=-9.2872 \times 10^{-3} \frac{\left(M_{1}+M_{2}\right)^{2 / 3}}{(P / 2 \pi)^{5 / 3}\left(1-e^{2}\right)},
$$

where $M_{1}$ and $M_{2}$ are in solar masses and $P$ is in days (Kopal 1959). Using the values given above, the theoretical relativistic contribution is $-0.05 \mathrm{yr}^{-1}$. Adding together the classical and relativistic contributions yields a total theoretical rate of change in the longitude of periastron as $-0.27 \mathrm{yr}^{-1}$, which is a little higher than our derived $-0.21 \mathrm{yr}^{-1}$.

The apsidal motion measurements and calculations should be taken as a first attempt to isolate and define these parameters. 
The period of precession of the MY Cyg system is roughly $1700 \mathrm{yr}$, of which only $33 \mathrm{yr}$ have been observed, or $2 \%$ of the cycle. A $2 \%$ interval on any part of a sine curve would certainly not begin to show the full shape. Hopefully, MY Cyg will be revisited at decade intervals to monitor the motion and provide a more complete understanding of the orbit.

\section{METALLICITY}

Popper (1971) noted that the MY Cyg spectra show Amstar features. Although he did not indicate from which star these lines are attributed, Kitamura \& Kondo (1978) stated both components are Am stars. Pols et al. (1997) compared the observed masses and radii of 49 detached, double-lined eclipsing binaries with their evolution models. Their maximum value of tested metal abundances was $Z=0.033$, and they indicated this was too low for MY Cyg. Ribas et al. (2000), in their study of the chemical composition of eclipsing binaries, took the MY Cyg values listed in Andersen (1991) but re-computed the temperatures, also obtaining values in the 7000-7100 K range for both stars. Tests with stellar evolution models were performed by Young et al. (2001), and they concluded MY Cyg is "underluminous relative to the models" and that a "higher heavy-element abundance would remove the discrepancy."

The WD program does not have an Am-star input parameter, but one can vary the ratio of metals-to-hydrogen $[\mathrm{M} / \mathrm{H}]$. The WD program allows only specific values of the metal ratio because of its use of prefitted Legendre functions to atmosphere models by Kurucz (1993). The $[\mathrm{M} / \mathrm{H}]$ parameter, which has as its reference the sun, differs from $Z$, the fraction by weight of all elements heavier than hydrogen and helium, and only $Z$ values were utilized in the studies described in the previous paragraph. At our request, D. J. Bord (2008, private communication) computed metal ratios for a few Am-star models with $Z$ in the range $0.02-0.06$. His corresponding $[\mathrm{M} / \mathrm{H}]$ values ranged from 0.0 to +0.5 . We re-ran the WD program with $[\mathrm{M} / \mathrm{H}]$ values of $0.0,+0.1,+0.2,+0.3$, and +0.5 . Our solution in Section 3 used the standard solar value of $[\mathrm{M} / \mathrm{H}]=0.0$, and our results did not change until $[\mathrm{M} / \mathrm{H}]=+0.5$ was tried. Given that our photometric data were obtained in the broad $U B V$ filter set, the lack of sensivity to the various $[\mathrm{M} / \mathrm{H}]$ values was not surprising.

\section{MAGNITUDES AND EVOLUTION}

The $U B V$ magnitudes for both components are listed in Table 5. These are based on $V=8.34, B-V=0.28$, and $U-B=0.14$ (outside eclipse) from Tremko et al. (1978), and on the relative ratios of light per bandpass as obtained with the WD program and light curve solution (see Table 4). The color indices from Tremko et al. did not change as a function of phase, and our magnitudes are not dereddened. The Hipparchos parallax is $0.00379 \pm 0.00087$ arcsec, or a distance of $264 \pm 63$ pc (ESA 1997). Using the apparent magnitudes and distance, the $V$ absolute magnitudes for each star are approximately $+2.0 \mathrm{mag}$. These are a little brighter than the $M_{\text {bol }}$ values in Table 5 .

The absolute dimensions do not match well with mainsequence values from Cox (2000). The masses are in the mid-tolate A star range, whereas the surface temperatures are typical for F2 stars (which we chose). The dilemma is resolved by the $2.2 R_{\odot}$ radii. With the aid of the HR Diagram from Sowell et al.
(2007; their Figure 2), it is seen that the MY Cyg components are evolved off the main sequence, at approximately luminosity class IV. This ties back to the discussion in Section 3 that Am stars have a galactic distribution similar to early $\mathrm{F}$ dwarfs and giants (Caballero-Nieves et al. 2007). Young et al. (2001) concluded MY Cyg is "well into main-sequence hydrogen burning" and assigned an age of 1.3 billion years. Using the isochrones of Girardi et al. (2000) for $Z$ of 0.019 and 0.030 , we posit a slightly younger age of 0.9-1.1 billion years.

This research has made use of the SIMBAD database, operated at CDS, Strasbourg, France. We thank Walter Van Hamme for advice regarding the WD program and for computing the error-bar values of the final solution parameters. We are indebted to Donald Bord for the Z-to-metal ratio computations.

\section{REFERENCES}

Andersen, J. 1991, A\&AR, 3, 91

Caballero-Nieves, S. M., Sowell, J. R., \& Houk, N. 2007, AJ, 134, 1072 Coughlin, J. 2007, undergraduate thesis, Emory Univ.

Cox, A. N. 2000, in Allen's Astrophysical Quantities, ed. A. N. Cox (4th ed; New York: Springer), 388

ESA 1997, The Hipparcos and Tycho Catalogues (Noordwijk: ESA) (ESA SP-1200)

Filatov, G. 1963, Byul. Inst. Astrofiziki, 35, 35

Gasposchkin, S. 1953, Harvard Ann., 113, 105

Girardi, L., Bressan, A., Bertelli, G., \& Chiosi, C. 2000, A\&AS, 141, 371

Guinan, E. F., \& Maloney, F. P. 1985, AJ, 90, 1519

Hardie, R. H. 1962, in Astronomical Techniques, Vol. 2, Stars and Stellar Systems, ed. W. A. Hiltner (Chicago: Univ. Chicago Press), 178

Hauck, B. 1994, in ASP Conf. Ser. 60, The MK Process at 50 Years, ed. C. J. Corbally, R. O. Gray, \& R. F. Garrison (San Francisco, CA: ASP), 157

Hauck, B., \& Curchod, A. 1980, A\&A, 92, 289

Hoffmeister, C. 1930, Astron. Nachr., 240, 195

Horak, J. 1966, Bull. Astron. Inst. Czech., 17, 27

Irwin, J. 1962, in Astronomical Techniques, Vol. 2, Stars and Stellar Systems, ed. W. A. Hiltner (Chicago: Univ. Chicago Press), 584

Jeffery, C. S. 1984, MNRAS, 207, 323

Johnson, H., \& Morgan, W. 1953, ApJ, 117, 313

Kitamura, K., \& Kondo, M. 1978, Ap\&SS, 56, 341

Kopal, Z. 1959, Close Binary Systems (New York: Wiley)

Kurucz, R. L. 1993, in Light Curve Modeling of Eclipsing Binary Stars, ed. E. F. Milone (New York: Springer), 93

Lucy, L. B. 1967, AZh, 65, 89

Pols, O. R., Tout, C. A., Schröder, K.-P., Eggleton, P. P., \& Manners, J. 1997, MNRAS, 289, 869

Popper, D. 1969, Bull. Am. Astron. Soc., 1, 257

Popper, D. 1971, ApJ, 169, 549

Popper, M. D., \& Etzel, P. B. 1981, AJ, 86, 120

Ribas, I., Jordi, C., Torra, J., \& Giménez, A. 2000, MNRAS, 313, 99

Rugemer, H. 1932, Astron. Nachr. Beobachtungs-Zirkular, 14, 46

Sowell, J. R., Tripp, M., Caballero-Nieves, S. M., \& Houk, N. 2007, AJ, 134, 1089

Sterne, C. E. 1939, MNRAS, 99, 451

Tremko, J., Papousek, J., \& Vetesnik, M. 1978, Astronomical Institute of the Slovak Academy of Sciences, 159, 199

Van Hamme, W. 1993, AJ, 106, 2096

Van Hamme, W., \& Wilson, R. E. 1984, A\&A, 141, 1

Van Hamme, W., \& Wilson, R. E. 1985, Ap\&SS, 110, 169

Van Hamme, W., \& Wilson, R. E. 2003, in ASP Conf. Ser. 298, GAIA Spectroscopy, Science and Technology, ed. U. Munari (San Francisco, CA: ASP), 323

Wachmann, A. A. 1948, Atron. Nachr. Erganzungshefte: Astron. Abhandlungen, $11, \mathrm{E}-40$

Williamon, R. M. 1975, AJ, 80, 976

Wilson, R. E. 1979, ApJ, 234, 1054

Wilson, R. E. 1990, ApJ, 356, 613

Wilson, R. E., \& Devinney, E. J. 1971, ApJ, 166, 605

Young, P. A., Mamajek, E. E., Arnett, D., \& Liebert, J. 2001, ApJ, 556, 230

Zessewitsch, V. P. 1954, Odessa Izv., 4, 268 